\section{Indirekte Krankheitskosten}

Helga Peter ${ }^{1}$ und Thomas Penzel ${ }^{2}$

${ }^{1}$ Marburg, Deutschland

${ }^{2}$ Interdisziplinäres Schlafmedizinisches Zentrum, Charité Universitätsmedizin Berlin, Berlin, Deutschland

\section{Englischer Begriff}

indirect costs of illness

\section{Definition}

Kosten, die durch eine Krankheit entstehen und volkswirtschaftlich gesehen einem Verlust von Arbeitspotenzial entsprechen. Es wird dabei unterstellt, dass aus volkswirtschaftlicher Sicht jede Gesundheitsausgabe eine Investition zur Erhaltung der Berufsfähigkeit des Patienten ist, sog. Humankapitalansatz. Das Humankapital wird durch krankheitsbedingte Fehlzeiten, eingeschränkte Leistungsfähigkeit oder vorzeitige Beendigung der Berufstätigkeit reduziert.

Siehe auch $\triangleright$ „Pharmakoökonomie“. 